\title{
CONCEPCIONES DE ESTUDIANTES Y PROFESORES ACERCA DE LA ENERGÍA DE LAS ONDAS
}

\author{
WELTI, REINALDO \\ Taller de Investigación en la Didáctica de las Ciencias. Departamento de Física y Química \\ Facultad de Ciencias Exactas e Ingeniería. Universidad Nacional de Rosario \\ Av. Pellegrini, 250. 2000 Rosario. Argentina
}

\begin{abstract}
Resumen. El propósito de este trabajo es examinar algunas dificultades que tienen estudiantes y profesores para interpretar y describir los mecanismos físicos asociados con la generación y propagación de una onda y la energía involucrada en estos procesos. Se analizan resultados de encuestas y entrevistas realizadas a docentes y alumnos de un curso introductorio de física de nivel universitario y se los compara con enunciados de libros de texto que son, o bien claramente incorrectos, o bien ambiguos y más o menos compatibles con los pensamientos de los estudiantes. Se consideran posibles causas de la difusión de estas ideas entre los que participan en el proceso de enseñanza-aprendizaje de las ciencias, y se esbozan algunas pautas para lograr un aprendizaje significativo de la temática analizada.
\end{abstract}

Palabras clave. Conceptualización, energía, ondas, dificultades de aprendizaje, enseñanza universitaria.

Summary. The purpose of this paper is to examine some difficulties students and professors have to interpret and describe the physical mechanisms associated with the generation and propagation of a wave and the energy involved in these processes. Results of surveys and interviews carried out to teachers and students of an introductory Physics course of university level are analyzed and compared with text-book statements that are, either clearly incorrect, or ambiguous and more or less compatible with the thoughts of the students. They are also considered the possible causes of the diffusion of these ideas among the actors participating in the process of teaching-learning of the sciences and rules are given to reach a significant learning of the analyzed subject matter. Keywords. Conceptualization, energy, waves, learning difficulties, university teaching.

\section{INTRODUCCIÓN}

El modelo ondulatorio ocupa actualmente un lugar fundamental en la estructura conceptual de la física y constituye una de sus síntesis más ricas y fructíferas. Como modelo, permite encontrar una unidad explicativa de fenómenos sumamente diversos: los pulsos en cuerdas, el sonido, los fenómenos luminosos, las emisiones de una antena de radio, las ondas de materia, etc. Dado su evidente grado de dificultad, este modelo no puede ser abordado de modo completo en la enseñanza secundaria $\mathrm{y}$ ofrece problemas de aprendizaje incluso en el nivel universitario. Existen obstáculos para la comprensión cinemática del movimiento ondulatorio, aun en situaciones en apariencia simples, como el pulso que se propaga en una cuerda. Sin embargo, no sólo la interpretación cinemática y la representación espacio-temporal presentan problemas, sino también su tratamiento dinámico.
Para avanzar en la elaboración de propuestas didácticas que contribuyan a un aprendizaje significativo de esta temática, se hace necesario conocer los nudos de dificultad de los estudiantes. En las investigaciones previas sobre la enseñanza-aprendizaje de las ondas algunos autores han observado complicaciones con la propagación de las ondas (Maurines, 1992; Fernández et al., 1993), con la física de las ondas sonoras (Linder y Erikson, 1989; Linder, 1993) y con la descripción matemática de las ondas y su superposición (Grayson, 1996; Wittmann, 1998, mientras que otros autores (Utges y Paca, 1998; Utges, 1999) sistematizan los principales modelos de onda que tienen los estudiantes secundarios.

En estos trabajos se menciona la energía de la onda solamente de manera indirecta, para relacionarla con la 
fuerza con que se agita la cuerda en un extremo o con la amplitud de la onda que se propaga. Como la energía es uno de los conceptos más importantes asociados a una onda, nos pareció interesante tratar de individualizar los obstáculos conceptuales en el aprendizaje de la energía de las ondas.

La investigación que se describe en este trabajo se encuadra en el paradigma que Novak (1988) define como «aprendizaje constructivista». En este paradigma, que interpreta la ciencia como una construcción humana, se supone que el aprendizaje debe ser considerado como:

- un proceso de construcción en la mente de cada alumno;

- una construcción de significados por interacción entre los esquemas mentales propios y las características del medio de aprendizaje;

- un proceso de elaboración colectiva en el que se confrontan ideas, se intercambian argumentaciones, se negocian y consensuan significados (Vygotsky, 1989);

- un proceso centrado en el estudiante, quien, guiado y orientado por el docente, es protagonista de su propio aprendizaje (Gowin, 1981).

Resulta evidente que, desde este enfoque, las ideas previas o modelos alternativos constituyen un eje central del aprendizaje. Como se ha mostrado en numerosas investigaciones recientes, estos modelos se construyen sobre la base de criterios, modos de razonar, propósitos y valoraciones que, si bien suelen ser suficientes para enfrentar las exigencias de la vida cotidiana, difieren sustancialmente de las pretensiones de precisión, coherencia, objetividad y sistematicidad del conocimiento científico y actúan como verdaderas «barreras críticas» (Driver, 1989) u obstáculos epistemológicos (Bachelard, 1972) para la comprensión de ciertos dominios de la ciencia.

Se sabe, por otra parte, que este tipo de ideas son particularmente persistentes y que oponen una tenaz resistencia a ser abandonadas o reemplazadas por otras de carácter científico, en el proceso denominado «cambio conceptual». Dicho cambio o ruptura epistemológica es considerado crucial para lograr un aprendizaje sistematizado, propio del conocimiento científico (Champagne et al., 1980), por lo que se considera que cualquier bloqueo que lo impida se convierte en un serio obstáculo para el aprendizaje de las ciencias.

Por lo general, el enfoque ha estado centrado en el individuo que aprende y en las estrategias pedagógicas consideradas más adecuadas para hacer aflorar en la conciencia de los estudiantes las concepciones consideradas «erróneas» a fin de que las mismas se anulen o modifiquen a través de la confrontación significativa con las nociones consensuadas por la comunidad científica (Posner el al., 1982; Brown, 1992).

El problema puede ser también analizado desde la representación epistemológica que incluye la estructura con- ceptual de la disciplina a ser enseñada, como una de las variables relevantes en el proceso de construcción del conocimiento por parte del individuo (Agnes, 1995; Hammer, 1996). Desde esta perspectiva, Viennot (1995) sostiene que revisar contenidos físicos y tratar de descubrir ideas y modos de razonamiento de los estudiantes son dos actividades mutuamente provechosas e incluso necesarias ambas si se espera algún progreso en el proceso de enseñanza-aprendizaje. En la misma línea de pensamiento, Lijnse (1994) califica como objetivo urgente de investigación el diseño de «estructuras didácticas» que describan contenidos y modos de enseñanza a escala relativamente pequeña (en oposición a la clásica investigación sobre currículo) y basándose en un cuidadoso análisis de las representaciones de los estudiantes. En cualquier caso, es necesario reconsiderar lo que tradicionalmente se enseña en cada dominio y debatir qué objetivos de enseñanza deberían adoptarse.

\section{CORPUS Y METODOLOGÍA}

Con este trabajo nos propusimos obtener información significativa sobre las concepciones de los estudiantes en un tema específico para tratar de optimizar el contenido y los modos de enseñanza. La investigación se ha llevado a cabo en una situación real en condiciones controladas sólo hasta donde la situación lo permitía. Por este motivo, puede considerarse que es, de acuerdo con Arnal (1992), una investigación evaluativa y cuasi experimental.

El proyecto surgió a partir de las evaluaciones y exámenes finales de alumnos que asistían a un curso de física básica en la Facultad de Ciencias e Ingeniería de la Universidad de Rosario, en las que comprobamos que, al expresar sus ideas sobre la energía de las ondas, los estudiantes hacían una transferencia inadecuada de los conceptos de la mecánica del punto a un medio continuo (Welti, 1998). Para precisar y definir en qué consistía el problema se diseñó un cuestionario (Anexo) con situaciones físicas relativamente simples, a partir de las que se pudiera aislar y describir dónde y por qué se producían las dificultades (Ary et al., 1987).

El cuestionario fue aplicado (en el año 1998) a estudiantes $(\mathrm{N}=67)$ que estaban cursando el tercer semestre de la carrera -todos habían hecho ya un curso sobre la mecánica del punto y asistían en ese momento a un curso de ondas-, y se tomó inmediatamente después de haber finalizado la presentación de la «cinemática» de la onda (velocidad de propagación, velocidad del medio, propagación de pulsos y de ondas armónicas).

Luego de relevar las respuestas, decidimos incorporar una estrategia cualitativa -la entrevista semiestructurada- para profundizar en algunos de los problemas detectados. Se realizaron así, a un grupo más reducido de alumnos $(\mathrm{N}=11)$, dos entrevistas individuales (grabadas y posteriormente transcriptas para su análisis, con una duración de 30 minutos cada una) que contribuyeron a depurar la lectura de los datos, interpretar las respues- 
tas y comprender aspectos del desarrollo secuencial del razonamiento llevado a cabo. Estos estudiantes fueron elegidos de acuerdo con un criterio aleatorio entre quienes describieron correctamente la cinemática del movimiento del elemento de cuerda (preguntas 1 y 2 del cuestionario) y dieron respuestas significativas a las preguntas referidas a la energía de las ondas $(3,4$ y 5$)$. No se amplió el número de alumnos entrevistados porque los razonamientos no tuvieron una gran dispersión y, además, porque los estudiantes seleccionados reunían las características apropiadas para el tipo de preguntas planteadas en las entrevistas (Samaja, 1993).

Dado que ésta era la primera vez que aplicábamos este tipo de cuestionario, nos pareció prudente repetirlo en las mismas condiciones al año siguiente $(\mathrm{N}=48,1999)$ para verificar si los resultados obtenidos medían realmente un patrón de razonamiento situado en una etapa particular de sus estudios. No se encontraron diferencias entre ambos grupos, salvo las atribuibles al azar (las diferencias de los porcentajes se mantuvieron inferiores al 5\%), de modo que, los datos estadísticos que se consignan en la próxima sección surgen de admitir que los dos grupos forman parte de una misma muestra.

Las formas de razonamiento estudiadas son compartidas, con frecuencia, por los profesores, los redactores de manuales y los especialistas, lo que produce una permanente retroalimentación de sus representaciones. Por este motivo, realizamos una revisión -que no pretende, de ninguna manera, ser exhaustiva- de un conjunto de textos que van desde un clásico que tiene más de cincuenta años, pasando por la bibliografía del curso y finalizando con páginas web. Analizamos, asimismo, las concepciones de un grupo de docentes $(\mathrm{N}=9,1998)$ que asistían a una sesión de perfeccionamiento sobre la física de las ondas para investigar en qué medida y de qué manera sus modos de razonamiento pueden resultar coincidentes con los del resto de los participantes del proceso de enseñanza-aprendizaje.

\section{ANÁLISIS}

\section{Analogía entre la energía de un MAS y la de una onda armónica}

El cuestionario fue preparado para explorar las ideas de los estudiantes acerca de la energía de una onda armónica que se propaga a lo largo de una cuerda. En particular, se pretendía averiguar las siguientes cuestiones:

- ¿Cómo describen el movimiento de un elemento de cuerda?

- ¿Cómo esquematizan estas ideas?

-¿Dónde suponen que la energía cinética y potencial son máximas (o mínimas) - ¿A qué interacción consideran responsable de la energía potencial del elemento de cuerda?

- ¿Cómo imaginan la distribución de energía a lo largo de la cuerda?
El análisis de los resultados nos permitió distinguir dos categorías de respuestas: las que describían correctamente la cinemática del movimiento del elemento de cuerda y las que no lo hacían. Todos los alumnos que describieron correctamente el movimiento del elemento de cuerda dieron respuestas significativas a las preguntas referidas a la energía de este elemento. Sin embargo, como era de esperar, los estudiantes que explicaron erróneamente la cinemática del movimiento del elemento de cuerda aportaron respuestas ambiguas o inclasificables.

Las respuestas se distribuyeron de la siguiente manera:

Preguntas 1 y 2: $\mathrm{El} 79 \%\left(\mathrm{~N}_{1}=91\right)$ muestra correctamente la posición del elemento de cuerda en cada una de las gráficas y concluye que efectúa un MAS. La respuesta del $21 \%\left(\mathrm{~N}_{2}=24\right)$ restante es poco clara, destacándose los que confunden el movimiento de un punto del medio con el movimiento de la onda.

El subgrupo $\mathrm{N}_{2}$ da respuestas dispersas y poco significativas a las restantes preguntas. A continuación los porcentajes se calcularán sobre el grupo $\mathrm{N}_{1}$.

Pregunta 3: El $96 \%$ contesta que la energía cinética es máxima en las gráficas 1,3 y 5 .

Pregunta 4: El $94 \%$ afirma que la energía potencial es máxima en las gráficas 2 y 4 . El $6 \%$ sostiene que la energía potencial es máxima en la gráfica 2 porque en ella el elemento de cuerda está en su máxima altura (¿tiro vertical?).

Pregunta 5: El $69 \%$ declara que la energía total en un MAS se conserva y (como en las gráficas 2 y 4 ) la energía cinética es nula, la energía potencial debe ser máxima. El $31 \%$ manifiesta que la energía potencial del elemento de cuerda es máxima cuando ésta se encuentra a la máxima distancia de la posición de equilibrio.

Pregunta 6: El 98\% de los estudiantes que afirma que, en las gráficas 1,3 y 5 , la energía cinética del elemento $P$ es máxima y que la energía potencial es máxima en las gráficas 2 y 4 , responden ahora que la energía cinética es máxima en los puntos $\mathrm{B}$ y $\mathrm{D}$ y que la energía potencial es máxima en los puntos A, C y E.

\section{La naturaleza de la energía potencial}

Uno de los temas más debatidos durante las entrevistas fue la naturaleza de la energía potencial y, en particular, cuál es la fuerza de interacción que la origina. El 81\% (9 sobre 11) de los entrevistados expresó que el elemento de cuerda efectúa un movimiento armónico simple y que, por lo tanto, al igual que en un oscilador a resorte que realiza este movimiento, se debe conservar la energía de cada elemento de la cuerda. Suponen que la energía potencial está relacionada con el trabajo que hay que realizar para sacar el elemento de cuerda de su posición de equilibrio y que, en consecuencia, la energía potencial es máxima cuando el elemento de 
cuerda está más lejos de su posición de equilibrio. Este razonamiento es consistente con la dinámica del MAS. Los estudiantes afirmaron que la energía potencial se debe al trabajo que hay que realizar para separar el elemento de cuerda de su posición de equilibrio; es decir, deducen la existencia de una especie de resorte que tiende a llevar al elemento de cuerda a su posición de equilibrio. En este caso, la energía potencial se acumularía en un resorte inexistente que está fuera de la cuerda. El 19\% restante opinó que la energía potencial de la cuerda es de naturaleza gravitatoria.

¿Dónde se almacena la energía potencial? Desde la física, el análisis es inmediato. Un elemento de cuerda no mantiene su longitud constante durante la oscilación. Su estiramiento (o deformación) es máximo cuando pasa por la posición de equilibrio (máxima pendiente de la cuerda) y nulo cuando su desplazamiento respecto del equilibrio es máximo (pendiente de la cuerda nula). El trabajo realizado por la tensión de la cuerda para producir este estiramiento se almacena en el elemento de cuerda como energía potencial y se devuelve al sistema cuando la longitud se contrae a su longitud natural. Sin embargo, ninguno de los entrevistados mencionó una posible conexión entre la energía potencial de la cuerda con el estiramiento de la misma.

En la segunda entrevista, se cambió la cuerda por una sucesión de masas y resortes y se les volvió a preguntar dónde se almacena la energía potencial. Los estudiantes encontraron enseguida una explicación: la energía potencial está relacionada con los estiramientos de los resortes. Sin embargo, cuando volvían a la cuerda, no pensaban que ésta se comportaba como una cadena de masa y resortes: «[...] la cuerda no se estira ni se comprime, la cuerda es inextensible», afirmaban. A pesar de que con el modelo de resortes y masas no tenían problemas para relacionar la energía potencial con el estiramiento de los resortes, seguían pensando que la cuerda no se comporta de esa manera.

\section{La energía se propaga cambiando de forma}

Con referencia a la pregunta 6 todos los entrevistados afirmaron que la energía cinética es máxima en los puntos B y D y que la energía potencial es máxima en los puntos A, C y E. Durante las entrevistas, defendían este punto de vista y utilizaban como principal argumento el siguiente: [...] «como la energía de la onda es en parte cinética y en parte potencial, ésta tiene que ir cambiando de forma [de cinética a potencial y viceversa] a medida que se propaga». Observemos que la respuesta a esta pregunta es en todo coherente con las dadas a las cinco primeras. Sus razonamientos pueden sintetizarse de esta manera: en un elemento de la cuerda, la energía de la onda pasa de energía potencial a energía cinética y de energía cinética a potencial, y así sucesivamente, y esta cadena de transformaciones que tiene una connotación cronológica en las cinco primeras preguntas, adquiere en la pregunta 6 un ordenamiento espacial en el que se alterna la energía cinética con la potencial.

\section{Relación entre la energía de la onda con el emisor que la origina}

Muchas de las ideas de la física de las ondas son sutiles y pocas veces están resaltadas de manera explícita en los textos. Por ejemplo, entender las ondas requiere comprender el rol de las condiciones iniciales para generarla. Los alumnos pueden tener dificultades para entender la propagación de la onda si no se hace una discusión suplementaria acerca de cómo son creadas. El trabajo de Maurines (1992) muestra que los estudiantes no pueden separar la propagación de la onda de las condiciones iniciales que describen su creación: en particular, suponen que la velocidad de propagación de un pulso depende de la forma en que la onda es generada por el emisor y no de las propiedades del medio.

Figura 1

Mecanismo interno del emisor y cuerda sujeta a la vara del emisor.

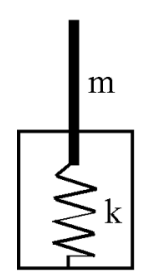

(a)

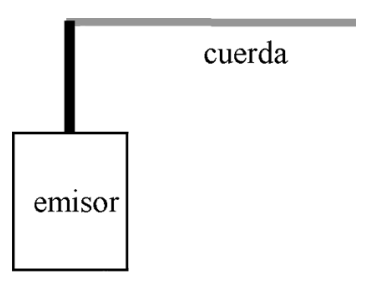

(b)
En nuestro estudio nos interesó particularmente investigar cómo se concibe el intercambio de energía entre el emisor que crea la onda y el medio de propagación. En las entrevistas presentamos una situación en la que se vincula el movimiento del emisor con el movimiento de la cuerda. El emisor es una caja en cuyo interior se encuentra el mecanismo que agita la vara en la cual está atada la cuerda (Fig. 1). En la figura 1a se muestra explícitamente el mecanismo que hemos elegido en nuestro caso: simplemente un resorte.

Con referencia a estas figuras se preguntó a los alumnos:

a) Si la vara de la figura 1a se comprime y luego se suelta, ¿qué tipo de movimiento realiza?

b) Si la vara, que ahora está unida a la cuerda como se muestra en la figura $1 \mathrm{~b}$, se comprime y luego se suelta, ¿qué tipo de movimiento realiza?

A la pregunta $a$, todos respondieron que, en ausencia de rozamiento, la vara realiza un movimiento armónico simple. La respuesta es físicamente correcta. Sin embargo, a la pregunta $b$, todos respondieron también, que la vara efectúa un movimiento armónico simple.

Los estudiantes insistían en que en ausencia de rozamiento la energía se debe conservar y, además, centraban su atención exclusivamente sobre el emisor omitien- 
do el movimiento de la cuerda. Para inducirlos a tenerlo en cuenta se les preguntó,

$P:$ «Qué le ocurre a la cuerda?»

$R:$ «a cuerda comienza a moverse y se crea una onda que se propaga a lo largo de la misma.»

$P$ : «¿Los elementos de cuerda que comienzan a moverse adquieren energía cinética?»

$R: \ll$ Sí.»

$P:$ «Quién entregó esta energía a ese elemento de la cuerda?»

$R:$ «La energía para poner en movimiento a los elementos de cuerda es suministrada por el emisor.»

$P$ : «Si el emisor entrega energía a la onda, ¿su energía se mantiene constante?»

$R:$ «No; la energía del emisor debe disminuir.»

$P:$ «Entonces, «¿qué tipo de movimiento efectúa el emisor?»

$R$ : «El emisor efectúa un movimiento oscilatorio amortiguado.»

La mayoría de los estudiantes, a pesar de estar convencidos de que este razonamiento era lógico y que llegaban a un resultado correcto, se mostraban sorprendidos de reconocer una situación en la cual un oscilador (el emisor) sin rozamiento realizaba un movimiento amortiguado.

Se advierte que están fuertemente influenciados por lo que han aprendido en la mecánica de una partícula (en ausencia de rozamiento, la energía total de una partícula se mantiene constante), y tienen dificultades para comprender que, si la energía del emisor disminuye, es, no porque se disipa, sino porque se transfiere a la cuerda para crear la onda que se propaga a lo largo de la misma.

\section{La propagación de ondas y el razonamiento causal lineal}

A docentes que realizaban una sesión de entrenamiento sobre el tema de la física de las ondas $(\mathrm{N}=9$, Universidad de Rosario, 1998) se les entregaron los dos extractos siguientes del libro The Feynman Lectures on Physics, (Feynman et al., 1966) y se les pidió que los leyeran atentamente para luego explicar su contenido en forma oral y por medio de gráficos.

1) «La consecuencia más remarcable de las ecuaciones de Maxwell es que éstas contienen la explicación de la radiación de ondas electromagnéticas a largas distancias. La razón es aproximadamente la siguiente: supongamos que en algún lugar tenemos un campo magnético que está creciendo porque aumentó súbitamente la corriente que circulaba a lo largo de un hilo. La ecuación de Faraday nos dice que este campo magnético que se incrementa crea un campo eléctrico. Este campo eléctrico que está creciendo, de acuerdo a la ley de AmpèreMaxwell, genera a su vez un nuevo campo magnético y este campo magnético produce un nuevo campo eléctrico y así sucesivamente. De esta manera, los campos se

En $a$ se muestra cómo los estudiantes distribuyen espacialmente las energías cinética y potencial a lo largo de la cuerda y en $b$ y $c$ cómo los profesores distribuyen espacialmente las energías eléctrica y magnética y potencial y cinética de ondas electromagnéticas y acústicas, respectivamente.

$\mathrm{a}$
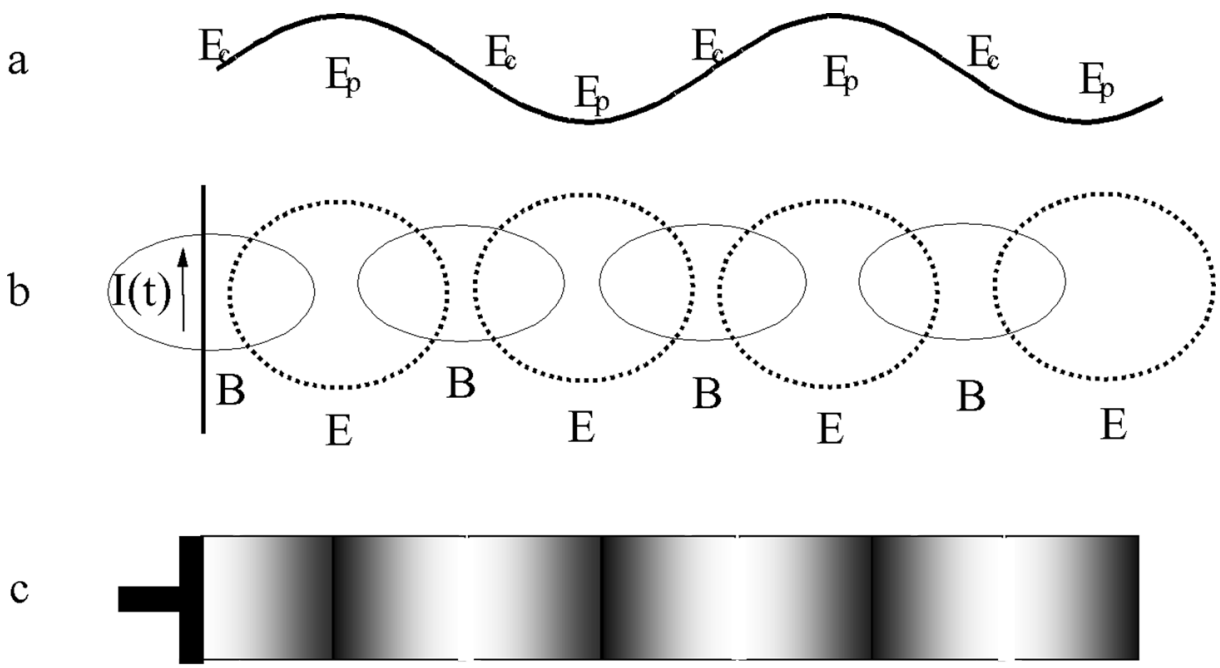

$\begin{array}{llllll}\mathrm{E}_{\mathrm{p}} & \mathrm{E}_{\mathrm{c}} & \mathrm{E}_{\mathrm{p}} & \mathrm{E}_{\mathrm{c}} & \mathrm{E}_{\mathrm{p}} & \mathrm{E}_{\mathrm{c}}\end{array}$ 
crean uno a otro a través del espacio sin necesidad de cargas y corrientes excepto en sus fuentes.»

2) «En la física de las ondas sonoras encontramos estos tres hechos característicos:

- El movimiento del aire provoca una variación de densidad,

- La variación de densidad produce una variación en la presión,

- Las variaciones de presión causan el movimiento del aire.»

Las gráficas que elaboraron 6 de los 9 profesores están resumidas esquemáticamente en la figura $2 \mathrm{~b}$ y $2 \mathrm{c}$. Observamos que ordenan sucesivamente en el espacio zonas de máximo campo magnético, seguidas de zonas de campo eléctrico máximo alrededor del hilo con corriente oscilante, y zonas de máximas variaciones de presión con zonas de velocidad de desplazamiento máximo en el interior del tubo donde se propaga una onda sonora.

Los docentes afirmaron que la energía magnética y la eléctrica se alternaban en el espacio: «[...] como la energía de la onda es en parte magnética y en parte eléctrica ésta tiene que ir cambiando de forma [de magnética a eléctrica y viceversa] a medida que se propaga». Notemos que este argumento es idéntico al que desarrollaron los estudiantes con respecto a la pregunta 6 del cuestionario.

Los enunciados analizados no son erróneos en sí mismos, pero han inducido, sutilmente, a cometer un error. Se puede percibir en ellos de manera subyacente una cadena causal entre las siguientes variables:

1)Corriente variable en el hilo $\rightarrow$ campo magnético variable en el tiempo $\rightarrow$ campo eléctrico variable en el tiempo $\rightarrow$ campo magnético variable en el tiempo, y así sucesivamente.

2) Movimiento del aire $\rightarrow$ variación de la densidad $\rightarrow$ variación de la presión $\rightarrow$ movimiento del aire, y así sucesivamente.

Esta cadena explicativa es la que Rozier (1987; Rozier y Viennot, 1991) denomina razonamiento causal lineal o razonamiento secuencial. En nuestro caso, la última de las variables coincide con la primera, componiendo lo que podemos denominar un razonamiento causal circular. En los enunciados presentados, la relación entre las variables es instantánea; sin embargo, es muy común interpretar las flechas horizontales no como «esto implica», o «por consiguiente», sino también como «después». La cadena explicativa adquiere, de esta manera, una relación cronológica y se transforma en un argumento del tipo historia:

1) La corriente variable crea primero un campo magnético, este campo magnético crea después un campo eléctrico, este campo eléctrico crea después un campo magnético, y así sucesivamente.
2)El movimiento de las moléculas del aire produce una variación de la presión, la variación de la presión produce después un movimiento del aire, este movimiento del aire produce después una variación de la presión, y así sucesivamente.

Esta connotación temporal adquiere en el problema que nos ocupa un ordenamiento espacial en el se alternan la energía magnética con la eléctrica, o la cinética con la potencial, como alumnos y profesores describen en la figura 2 .

Creemos que ésta es una de las causas por la cual, en el nivel de expertos (especialistas y docentes), está fuertemente instalado el modelo de una sucesión espacial entre los dos tipos de energía. Los razonamientos de los estudiantes, manifestados en los resultados de la encuesta, entran, por lo tanto, en resonancia con los de los profesores y se refuerzan.

\section{La energía de las ondas en textos y notas de clase}

Se observa (Viennot y Rozier, 1994; Welti, 2000) que, con frecuencia, las ideas de los estudiantes son ampliamente compartidas por todos quienes participan en el proceso de enseñanza-aprendizaje de las ciencias. En particular, numerosos libros de textos, documentos de divulgación y notas de clase se hacen eco de las concepciones e ideas de los estudiantes. Por lo tanto, hay un permanente refuerzo de sus ideas o al menos de su manera de ver las cosas.

No es de extrañar, entonces, que en la mayor parte de los materiales escritos que hemos examinado se encuentren razonamientos similares a las respuestas que dan los estudiantes a la encuesta que realizamos. Sin embargo, se pueden distinguir diferentes niveles de compatibilidad entre las ideas comunes y lo que expresan los textos. Algunos comentarios que a primera vista no conllevan ninguna consecuencia pueden tener efectos determinantes si se le agregan componentes como los descritos en la sección anterior. Otros son francamente erróneos o bien ambiguos y más o menos compatibles con las ideas de los estudiantes. A continuación presentamos algunos de estos enunciados.

Borowitz y Bornstein (1968) afirman que «cada partícula de la cuerda tiene un movimiento armónico simple. Hemos visto que en un movimiento armónico simple, la energía del oscilador se convierte continuamente de energía cinética en elástica y viceversa. Entonces, cuando el elemento de cuerda está momentáneamente en reposo, no tiene energía cinética y toda su energía es elástica».

Observamos que este razonamiento es idéntico al que hacen nuestros estudiantes, y coincide con lo que afirma Watson (1950): «[...] cuando un punto de la cuerda está en su máxima elongación, a uno y otro lado de su posición de equilibrio, queda un instante en reposo, y toda la energía se encuentra en estado potencial debido a la deformación de la cuerda. Cuando el elemento de 
cuerda pasa por su posición de equilibrio, toda su energía es cinética».

Lo mismo ocurre en Tipler (1995): «Si consideramos a cada elemento de cuerda de masa $x$ como un oscilador armónico simple cuya máxima amplitud es A, sabemos que su energía total será $E=1 / 2 \omega^{2} A^{2} D x$, donde $\omega$ es la frecuencia de la onda.

En otros textos, como el de Arons (1970), el comentario es ambiguo, resultando más o menos compatible con las ideas de los estudiantes: «[...] cualquier región pequeña del espacio que es alcanzado por una perturbación ondulatoria posee energía cinética. El sistema como un todo también tiene energía potencial que depende de la posición de la región en cuestión. En una cuerda que vibra transversalmente, la energía potencial está asociada con los desplazamientos en contra del efecto de restitución debido a la tensión de la cuerda».

En algunos textos -que tratan correctamente el tema-se advierte que el resultado no es intuitivo ni evidente. Por ejemplo en Roederer (1966): «[...] en una porción del medio, en la que en un instante dado la densidad de energía cinética es máxima, también la densidad de energía elástica será máxima. Esto aparenta ser un contrasentido, pero se explica observando la figura [...]». El autor remite a una figura en la que se muestra que, cuando la cuerda pasa por su posición de equilibrio, su velocidad es máxima y también su deformación (estiramiento) es máxima.

En el curso de Mestre y otros (1998) se utiliza el siguiente argumento para explicar como «viaja» la energía en una onda:

«Hemos examinado cómo la energía de un cuerpo se transforma de una forma a la otra (potencial y cinética) como ocurre en el ejemplo del péndulo. Ahora vamos a aplicar estos conceptos a las ondas.»

Cuando una onda viaja a través de un medio, la propiedad física que se propaga a través del medio es energía. Para las ondas en cuerdas o las ondas sonoras, esta energía que se propaga debe estar en la forma de energía cinética y potencial. En muchos casos, la energía de la onda es una mezcla de energías cinética y potencial, a pesar de que la naturaleza de esta mezcla varía con el tiempo.

Un modelo que muestra cómo viaja la energía a través de un medio y al mismo tiempo cambia su forma de energía cinética a potencial y, viceversa, es una cadena de masas conectadas entre sí por resortes sin masa. En la figura 3 se muestra una serie de dibujos que muestran a un pulso longitudinal que se propaga a lo largo de la cadena.

«Si despreciamos la fricción, la energía total permanece constante, a pesar de que está constantemente cambiando de forma. En las líneas 1, 3, 5... la energía es toda cinética; en $2,4,6 \ldots$ es toda potencial.»

En estos enunciados se advierte con claridad cómo la suposición errónea de que la energía potencial y cinética
Figura 3

Modelo para estudiar cómo viaja la energía a través de un medio.

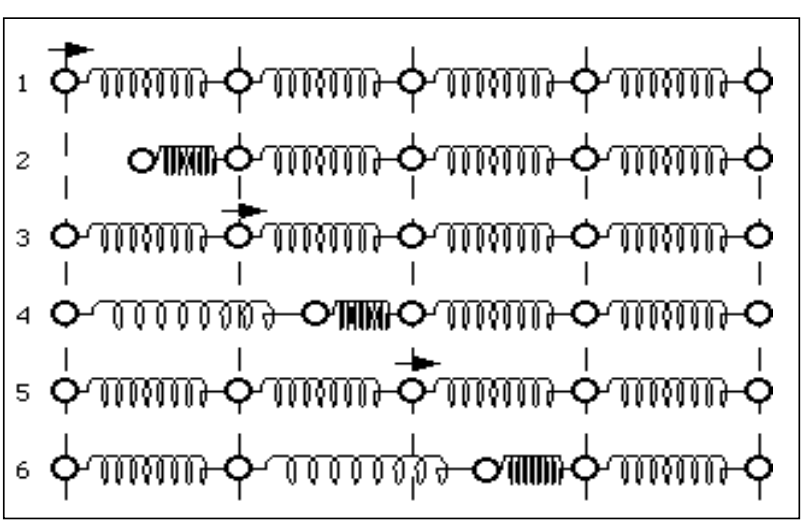

se alternan en el tiempo (en un elemento del medio en el que se propaga una onda) induce a pensar que éstas también se alternan espacialmente, y se llega a una explicación que coincide con la de nuestros estudiantes y docentes.

El modelo que proponen Mestre y otros (1998) puede ser adecuado para que los estudiantes relacionen la energía potencial con la deformación del medio como mencionamos anteriormente. Sin embargo, este modelo sólo puede utilizarse cuando la longitud característica del pulso es mucho mayor que la longitud de uno de los elementos masa-resorte.

\section{COMENTARIOS FINALES}

Los resultados alcanzados en nuestra investigación nos permiten adelantar algunas interpretaciones del razonamiento que realizan los estudiantes con respecto al tema tratado. En primer término, descubrimos que hay una interacción entre el nuevo conocimiento (energía de la onda) y el conocimiento previo (oscilador armónico) y, en consecuencia, se aplican significados anteriores a la nueva situación planteada. Se indica que el elemento de cuerda realiza un movimiento armónico simple (MAS) similar al del oscilador de resorte estudiado en el semestre anterior. Después de haber encontrado esta similaridad «cinemática», los alumnos deducen que las propiedades dinámicas del elemento de cuerda son las mismas que las de un oscilador de resorte. En particular, concluyen: $a$ ) que la energía del elemento de cuerda se conserva; y $b$ ) que su energía potencial es máxima cuando su elongación es máxima y, en esa posición, su energía cinética es cero.

Es posible observar que el problema de la cuerda -que pertenece a la mecánica del continuo- es tratado como si fuese una prolongación de la mecánica del punto y se presume que, en un sistema de partículas que interactúan entre sí, la conservación de la energía del sistema implica la conservación de la energía de cada una de ellas. Por 
lo tanto, no se entiende que, cuando la energía de una de ellas disminuye, no es porque haya pérdidas, sino porque se la transfiere a otra y tampoco se logra interpretar el mecanismo de transporte de energía que está asociado a la propagación de una onda. Lo mismo ocurre con la transferencia de energía desde el emisor al medio de propagación cuando se crea la onda.

Comprobamos que hay una tendencia a analizar las preguntas recurriendo a modelos semejantes a los denominados, por Utges (1999), repetición y sucesión. En estos modelos, cada punto de la cuerda es pensado como un oscilador aislado que no interactúa con sus vecinos. Los estudiantes advierten que lo que ocurre en un punto puede ocurrir después en otro punto, pero no reconocen el vínculo que explica la transferencia de la energía. Esto los lleva a imaginar una conservación local de la energía, propia de un oscilador armónico, pero incorrecta para interpretar el fenómeno que se les está proponiendo.

Al confundir, además, el desplazamiento con la deformación del elemento de cuerda, infieren que el máximo de energía potencial corresponde al máximo desplazamiento de la cuerda. Más aún, aunque en las entrevistas se les sugirió que examinaran un modelo de masas y resorte, afirmaron que la cuerda era indeformable y no admitieron su elasticidad: como la cuerda no es un resorte, no se estira. Esta propensión a suponer que hay cuerpos que tienen la propiedad de ser indeformables ha sido encontrada también por Di Sessa (1992), en sus entrevistas con alumnos de física, con quienes analizaba el rebote de un objeto que caía sobre el piso.

Para superar algunos de los obstáculos conceptuales en el aprendizaje de la energía de las ondas, consideramos conveniente introducir, antes de iniciar el estudio del transporte y almacenamiento de energía, algunos contenidos de la mecánica del continuo que permitan escribir explícitamente la ecuación de conservación de la masa y energía. Esta propuesta coincide con la formulada por
Herman (2000) y su grupo de trabajo de la Universidad de Karlsruhe, que sugieren, adoptando un modelo de sustancia para las magnitudes extensivas, establecer una ecuación de balance entre el flujo de estas magnitudes a través de una superficie cerrada y la velocidad con que varía ésta en el volumen encerrado por dicha superficie. La jerarquización de los principios más abarcadores facilita la formulación de la estructura conceptual de la física y posibilita una acomodación del pensamiento científico al conocimiento intuitivo de los alumnos.

La mayoría de los textos que estudian la energía de las ondas, sin emplear las ecuaciones del balance de la masa y energía en un volumen de control, se valen, al igual que nuestros estudiantes, de la analogía entre el movimiento de un elemento de cuerda con un sistema masa resorte para concluir que la energía potencial y la cinética se alternan en el tiempo y que la energía potencial es máxima cuando la separación del elemento de su posición de equilibrio es máximo. No es de extrañar, por lo tanto, que los profesores que se formaron leyendo estos textos tengan ideas concordantes con las de los estudiantes. En este contexto se genera una retroalimentación permanente de estas ideas.

Es necesario que los docentes adoptemos una actitud vigilante y activa para que nuestros estudiantes no reemplacen las ideas científicas por las comunes. Tenemos que identificar los enunciados incorrectos y descubrir los comentarios ambiguos que son más o menos compatibles con las ideas comunes. Hay diversos modos posibles de trabajo como, por ejemplo, utilizar cuestionarios similares a los utilizados en nuestras encuestas y entrevistas como ejercicios cualitativos para crear una situación de conflicto o realizar otras actividades, como la crítica guiada de textos. Actualmente estamos implementando algunas de estas propuestas en nuestros cursos y hemos podido comprobar que resultan sumamente útiles para alcanzar una comprensión significativa de esta temática.

\section{REFERENCIAS BIBLIOGRÁFICAS}

AGNES, C. (1995). Physical laws revisited. Thinking Physics for teaching. Bernardini, Tarsitani and Vicenti (ed.). Nueva York: Plenum Press.

ARNAL, J., DEL RINCÓN, D. y LATORRE, A. (1992). Investigación educativa: fundamentos y metodologías. Barcelona: Labor, SA.

ARY, D., JACOBS, L.C. y RAZAVIEH, A. (1987). Introducción a la investigación pedagógica. México: Interamericana.
ARONS, A.B. (1970). Evolución de los conceptos de la física. México: Trillas.

BACHELARD, G. (1972). La formación del espíritu científico. Buenos Aires: Siglo XXI.

BOROWITZ, S. y BORNSTEIN, L.A. (1968). A Contemporary View of Elementary Physics. Tokyo: McGraw-Hill Book Company. 
BROWN, D.E. (1992). Using examples and analogies to remediate misconceptions in Physics: Factors influencing conceptual change. Journal of Research in Science Teaching, 29(1), pp. $17-34$.

CHAMPAGNE, A.B., KLOPFER, L.E. y ANDERSON, J.H. (1980). Factors influencing the learning of classical mechanics. American Journal of Physics, 48, pp. 1074-1079.

DI SESSA, A.A. (1992). Phenomenology and the Evolution of Intuition, en Getner, D. y Stevens, A.L. (ed.).Mental Models, pp. 15-33. Hillsdale, Nueva Jersey: Lawrence Erlbaum Associates.

DRIVER, R. (1989). Changing conceptions. Adolescent development and school science. Adey, P. (ed.). Londres: Falmer Press.

FERNÁNDEZ, P., JARDÓN, A. y UTGES, G. (1993). Propagación de pulsos en cuerdas. Una aproximación al modelo espontáneo de los alumnos. VIIIReunión de Educadores de Física (REF VIII). Rosario, Argentina.

FEYNMAN, R.P., LEIGTHON, R.B. y SANDS, M. (1966). The Feynman Lectures on Physics. Nueva York: Addison Wesley.

GOWIN, D.B. (1981). Educating. Ithaca, Nueva York: Cornell University Press.

GRAYSON, D. J. (1996). Using education research to develop waves courseware. Computational Physics, 10(1), pp. 3037.

HAMMER, D. (1996). More than misconceptions: Multiple perspectives on student knowledge and reasoning, an appropriate role for education research. American Journal of Physics, 64(10), pp. 1316-1325.

HERRMANN, F. (2000). The Karlsruhe Physics Course. European Journal Physics, 21, pp. 49-58.

LIJNSE, P.L. (1994). Second taught on a summer school. European Research in Science Education. Proceedings of the first PhD Summer School, pp. 341-357. Utrech.

LINDER, C. J. y ERICKSON, G. L. (1989). A study of tertiary physics students' conceptualizations of sound. International Journal of Science Education, 11(5), pp. 491-501

LINDER, C.J. (1993). University physics students' conceptualizations of factors affecting the speed of sound propagation. International Journal of Science Education, 15(6), pp. 655-662.

MAURINES, L. (1992). «Spontaneous reasoning on the propagation of visible mechanical waves». International Journal of Science Education, 14(3), pp. 279-293.

MESTRE, J., MULLIN, W. J. y GERACE, W. J. (1998). Theory of Sound with Aplications to Speech and Hearing Science. Classnotes 8: Massachusetts University. (http://wwwunix.oit.umass.edu/ phys 114/Tutorial/ClassnotesIndex.html)
NOVAK, J. (1988). Learning Science and the science of learning. Studies in Science Education, 15, pp. 77-101.

POSNER, G. J., STRIKE, K. A., HEWSON, P.W. y GERTZOG, W.A. (1982). Accommodation of a scientific conception: towards a theory of conceptual change. Science Education, 66(2), pp. 211-227.

ROEDERER, J.G. (1966). Mecánica elemental. Buenos Aires: Editorial Universitaria de Buenos Aires.

ROZIER, S. (1987). «Le raisonnement linéaire causal en thermodynamique clasique élémentaire». Tesis doctoral. París: Universidad París 7.

ROZIER, S. y VIENNOT, L. (1991). Student's reasoning in thermodynamics. International Journal of Science Education, 13(1), pp.159-170.

SAMAJA, J. (1993). Epistemología y metodología. Buenos Aires: Eudeba.

TIPLER, P.A. (1995). Física. Barcelona: Reverté, SA.

UTGES, G. y PACCA, J. (1998). O que é una onda. Um estudo sistemático do conceito procurando subsidios para o ensino. $V$ Encuentro de Pesquisa em Ensino de Física (V EPEF), Florianópolis, Brasil.

UTGES, G. (1999). «Modelos e analogías na comprensâo do conceito de onda». Tesis doctoral. Universidad de San Pablo. Sao Paulo, Brasil.

VIENNOT, L. y ROZIER, S. (1994). Pedagogical Outcomes of Research in Science Education: Examples in Mechanics and Thermodynamics. The Content of Science: A Constructivist Approach to its Teaching and Learning, pp. 237-254. Fensham, P., Gunstone, R. y White, R. (ed.). Londres: The Falmer Press.

VIENNOT, L. (1995). The Contents of Physics. Essential Elements, Common Views. Thinking physics for teaching. Bernardini, Tarsitani and Vicenti (ed.). Nueva York: Plenum Press.

VIGOTSKY, L.S. (1989), El desarrollo de los procesos psicológicos superiores. Barcelona: Crítica.

WATSON, W. (1950). Curso de física. Barcelona: Labor, SA.

WELTI, R. (1998). Obstáculos conceptuales en el aprendizaje de la energía de las ondas. IV Simposio de Investigadores en Educación en Física, (SIEF 4). La Plata, Argentina.

WELTI, R. (2000). Conceptualizaciones de estudiantes universitarios de física sobre la energía de las ondas mecánicas. VII Encuentro de Pesquisa em Ensino de Física (VII EPEF). Florianópolis, Brasil.

WITTMANN, M. (1998). «Making sense of how students come to an understanding of physics. An example from mechanical waves». Tesis doctoral. Universidad de Maryland. EEUU.

[Artículo recibido en diciembre de 2000 y aceptado en octubre de 2001.] 


\section{ANEXO}

\section{Cuestionario}

Una onda armónica se propaga a lo largo de una cuerda, sin fricciones internas ni externas. En las cinco primeras gráficas de la figura se muestran instantáneas de la cuerda separadas por un intervalo de tiempo igual a $T / 4$ donde $T$ es el período de la onda.

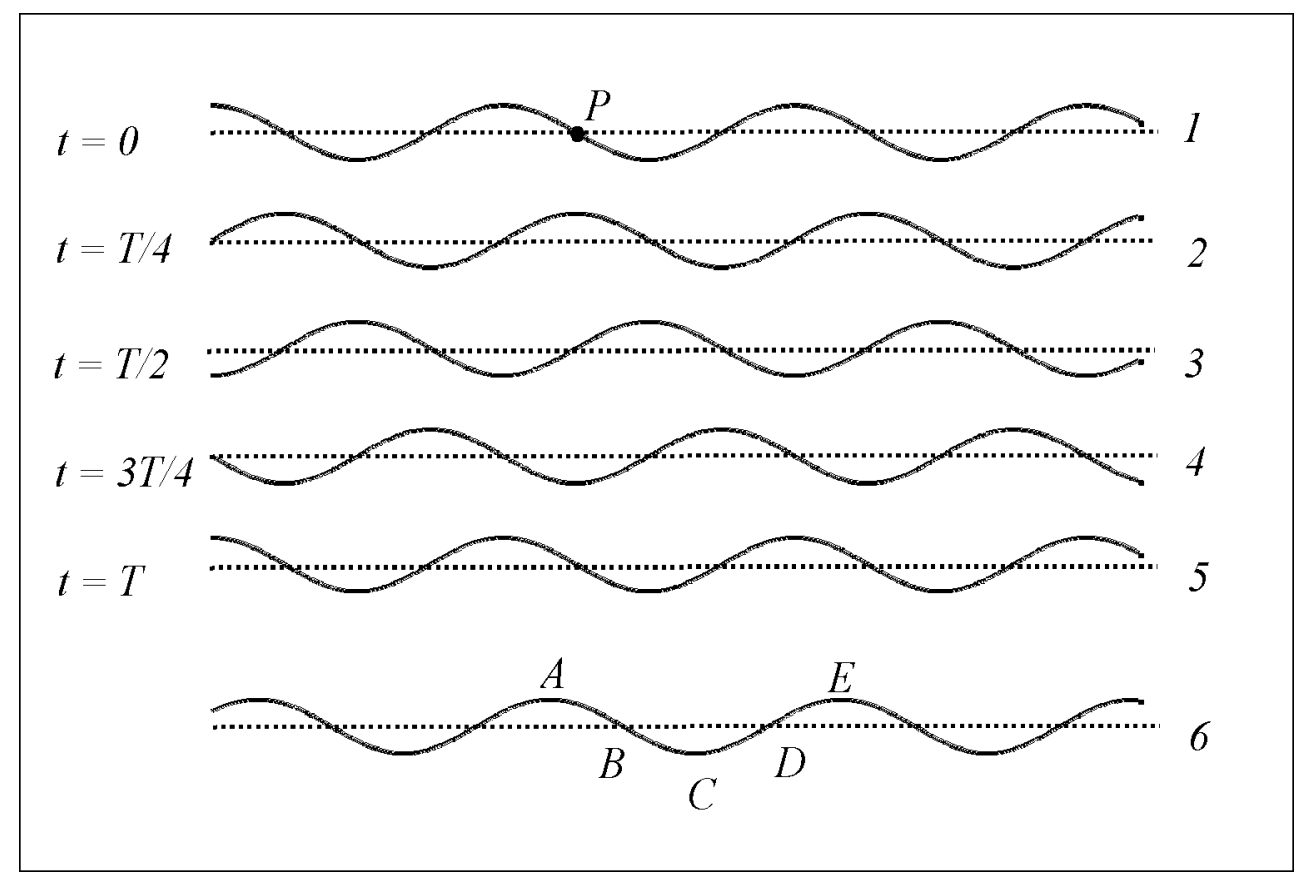

1) Muestre sobre las gráficas 2, 3, 4 y 5 la posición del elemento de cuerda $P$ que se muestra en la gráfica 1 .

2) Grafique el desplazamiento de $P$ en función del tiempo. ¿Qué tipo de movimiento realiza $P$ ?

3) ¿En cuál de las gráficas (1, 2, 3, 4 o 5) la energía cinética es máxima?

4) La cuerda tiene, además, energía potencial. ¿En cuál de las gráficas la energía potencial de $P$ es máxima?

5) Justifique la respuesta dada en el punto 4.

6) La gráfica 6 es una instantánea de la cuerda en un tiempo cualquiera. ¿En cuál de los puntos $A, B, C, D, E$, la energía cinética es máxima y en cuál de ellos la energía potencial es máxima? 